\title{
COMPUTATIONAL MOBILE ARCHITECTURES. PRACTICAL APPROACH
}

\author{
Marian Pompiliu CRISTESCU \\ Lucian Blaga University of Sibiu, Romania \\ marian.cristescu@ulbsibiu.ro \\ Mihai-Gabriel CRISTIAN \\ Lucian Blaga University of Sibiu, Romania \\ mihai.cristian@ulbsibiu.ro
}

\begin{abstract}
When designing architectures for Mobile Grid infrastructures and scenarios based on such infrastructures, the basic idea is to extend Grid computing systems in Mobile computing-based environments where mobile devices can be effectively embedded within the Grid - either as receivers / service recipients, or, more valuable, service providers. The paper presents a customized version of these types of architecture through several scenarios in different domains that integrate Grid and Mobile Grid technologies with industry-specific technologies, and then integrates the Mobile Grid architecture with P2P architectures.
\end{abstract}

Keywords: mobile architectures, Grid systems, P2P nodes, topologies.

JEL classification: C63, C88

DOI: $10.12948 / \mathrm{ie} 2019.06 .01$

\section{Introduction}

Distributed architecture can connect multiple "components" - into a hybrid system that integrates Grid technologies and Peer-to-Peer (P2P) technologies - for secure information sharing and data transfer between participants. Distributed computing technologies are used to connect static and mobile systems, integrating the various devices and devices, intelligence and intelligence analysis systems and databases to allow aggregation of the data taken and their integration into the virtual environment specific to the situation and context.

By combining Grid and P2P systems, an intelligently distributed and self-adaptable architecture can be created to radically change the amount of information available. The proposed P2G (Peer-to-Grid) framework strengthens P2P and Grid technologies, addressing the mobility of resources offered by transient devices in the context of the Web Services Resources Framework (WSRF) framework. This step towards a P2G hybrid architecture represents a change of vision and a new paradigm from two perspectives: firstly, this hybrid architecture addresses the uniform integration of mobile and static distributed resources by integrating Grid and P2P computational technologies, incorporating real mobility solutions and for the transient connectivity of the devices; Secondly, hybrid architecture allows integration of components and data at execution time through service-oriented and dynamic discovery of services and resources.

\section{Literature review and background}

The generic term "mobile computational technologies," implies according to [11], as diversified methods that call upon various mobile devices with varied constructive and functional features to allow access to information and communicate and transfer data, anywhere and at any time by using any available method. 
According to [14], the Mobile Grid concept is described as a set of services aimed at meeting the mobility requirements of static and mobile users, allowing them access to Grid resources through technologies implemented on the basis of transparency and efficiency.

In [1] the idea is that, following a process of transposing mobile grid mobility issues, it will be able to allow, on the one hand, the mobility of users who perform operations to access a static Grid, and another part also uses the mobility aspects of the resources that represent Grid components.

An approach to service mobility in terms of infrastructure capacity to provide mobile users with a diversified range of services over a defined time span to ensure service independence with regard to the devices they require and the location finds the user, all related to the network to which users have access, is presented in [2].

A Grid service is "a Web service which provides a set of well-defined interfaces and follows specific conventions for building applications in Grid environments", "a web service that conforms to grid computing standards", "a stateful web service with an associated lifetime which provides a set of interfaces through which grid users may interact", "a processing component in the Grid environment with the WSRF compliant Grid standard interfaces. They can invoke each other in the virtual organization" [7].

Virtualization of Grid services (similar to virtualization of Web services) is essential for running services at a higher level on heterogeneous device collections. In a Grid environment, virtual Grid services help provide a superior virtual layer across multiple Grid variants, making it possible to map / translate common semantic service functionality across multiple platforms [5].

Some authors [5], [12] describe the concept of Mobile Grid in close correlation with elements of infrastructure and Grid and computational mobile technologies used, and all of these are found in the process of describing and defining the term Grid, the process in which they occur, as helpful elements useful in the activity that aims to associate mobile users with mobile resources through actions characterized by uniformity, transparency, security and efficiency.

\section{Method description and terms presentation}

In designing architectures for Mobile Grid infrastructures and scenarios based on such infrastructures, the basic idea is to extend Grid computing systems to Mobile Computer based environments in which mobile devices can be effectively embedded within the Grid - either as receivers / service recipients, or, more valuable, service providers. These architectural variants can be developed and expanded in different ways to meet the particular requirements of different application areas and deployment scenarios. Expanding studies and research have customized these types of architecture for several scenarios in different areas, integrating Grid and Mobile Grid technologies with industry-specific technologies, and further integrating the Mobile Grid architecture with P2P architectures.

\subsection{Mobility of services and resources}

The resource mobility potential has raised the issue of resource discovery (WSRF does not specify how resources are discovered or created). If a resource is released from the endpoint / destination of the Web service and becomes essentially autonomous, resources can be discovered and rediscovered in a decentralized manner and, moreover, when it comes to mobile resources, it is possible to discover and rediscover resources were "lost". It is a necessary mechanism for discovering decentralized resources [3].

If the resource is moving and is still exposed by another Web service, the "resolver" entity will refer the consumer to another location on the network. It may be necessary for the "resolver" entity to be discovered, and then the resolver entity must discover the service that now has 
access to the resource to get the real address (Figure 1 - b). The "resolver" entity can be notified about the location of the service, or discover the new service alone.

\section{The proposed architectural variant}

Developments in the Grid technologies [2], [13] and Peer-to-Peer (P2P) [9], [8] allow research communities to explore exploring the possibilities for these systems to be adapted to improve the operating mode and current procedures and to provide new solutions to difficult issues that persist in our daily lives. There are many areas where such problems arise, although sometimes they are still difficult to solve, due to the distributed nature of the institutions involved. Distributed character is combined with very strict security requirements, on confidentiality of personal data, access to data and processing of personal data. That is why such infrastructures are very suitable areas of application to take advantage of the capabilities that a hybrid Grid/P2P hybrid infrastructure can offer [4], [6].

Based on these specifications, we have made a proposal for hybrid mobile architecture that we have presented below, in which we have indicated the use of static and dynamic Grid technologies in order to obtain a method of integrating the system components with some mobile agents seeking to streamline such interactions. We have been looking at how interactions between static Grid nodes and different mobile devices can be implemented, the type of action of which determines us to assimilate them as mobile Grid nodes to design, develop and implement the software capabilities involved in building a bridge at these levels two levels or environments.

These entities must be independently connected and integrated, and the interconnection method involves the realization and implementation of a bridge component that we have called the "gateway" to which it has been developed the ability to conduct "negotiations" involving "actors "Such as mobile devices that are connected by wireless technologies on the one hand and the static Grid on the other.

A goal pursued at this stage was to ensure the "cache" of information - at the level of the identified entities, imposed by the transient character, plus the lack of safety of the mobile devices. The stated intention was to identify practical ways to secure and stable gateway components that can be used to store information.

The method used assumes that the mobile nodes send requests to the static Grid in which they request data storage or perform jobs that are involved in the process by performing computations with a high level of complexity. Components, such as the Globus Toolkit, included in the Grid middleware category, are designed and developed using static Grid resources, and will be interfaced with mobile Grids using gateway components whose basic feature is that they can ensure interoperability.

Another goal pursued was to store the information in the waiting queue when the affected components are disconnected. Thus, we have developed and deployed a generic Gateway infrastructure to enable interactions between mobile and static grids. In this regard, it has been established the need to create components that play the role of Mobile Agents, starting from the technologies that are based on such agents and some of the Mobile Queuing Server components. 


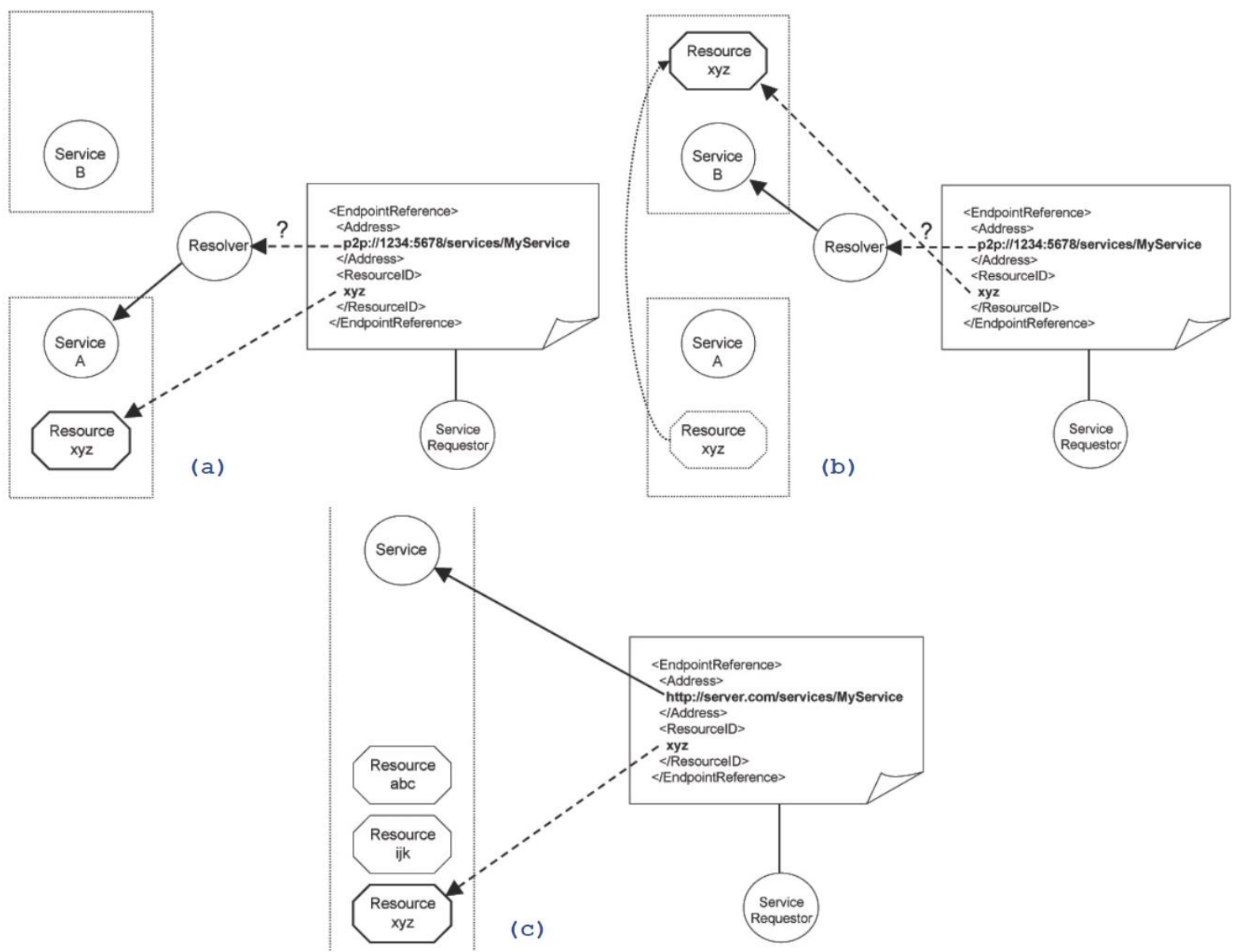

Figure 1. Localization of services and resources: (a) through logical addresses and the "solve" mechanism, (b) through logical addresses and the "solve" mechanism in mobility conditions, (c) without logical addresses and without the mechanism of "solving"

In order to be able to change transport protocols, while maintaining the connectivity provided by the proxy with the static Grid, an infrastructure has been developed that includes the gateway components and provides for the transient connection of the mobile nodes, allowing them to oscillate between the connected states and / or disconnected. This technique ensures the conservation of energy resources and involves limited connectivity to allow mobile nodes to perform or receive current updates in an optimal timeframe [10].

\subsection{Mobile Agents and Job-Proxy objects}

In the proposed architectural design, there are Mobile Agents (MA) components. Such components are placed on the Gateway level of the hybrid mobile architecture and play the role of an entity that will act as mediating agents that make it possible to include in the projected and developed infrastructure some transient mobile devices whose main limitations relate to the lack of stability.

For the design, development and implementation of an authorization method, Job-Proxy objects have been included in the proposed and developed architecture. Starting from mobileto-job mobile job request, using mobile agent technology, the MA component placed on Gateway develops a Job-Proxy object whose task is to resolve requests from the mobile user who the job was sent.

The mobile user can set the duration of a Job-Proxy expiration, for example, the job being executed on a node. The static grid will be canceled after a certain amount of time and all 
Gateway resources allocated for that Job-Proxy are released. This operation is required because a mobile user may not be able to explicitly request the job to be canceled even though the running job is no longer required. If the job is not automatically canceled, unnecessary job support continues to consume resources at both the Static Grid and the Gateway in the Mobile Grid infrastructure. This versatility of the Job-Proxy object is the result of the combination of several recent paradigms in programming: the capabilities called generic term "patterns" (such as "proxy pattern" and "delegation pattern") and the chain of responsibilities.

\subsection{The architectural components of the mobile node}

Based on the specific elements of the proposed hybrid mobile architecture and taking into account the characteristics of the framework WSPeer described in [15], we have presented in Figure 2, the components of the architecture of the mobile node. The mobile node contains a number of intercommunicating architectural components, which allows implementation of the functionalities required by the proposed architecture. These are:

- the service level;

- the SOAP module parses and generates SOAP messages;

- the data channel module;

- the migration module;

- the P2P module;

- the device module;

- the protocol module;

- the transport module.

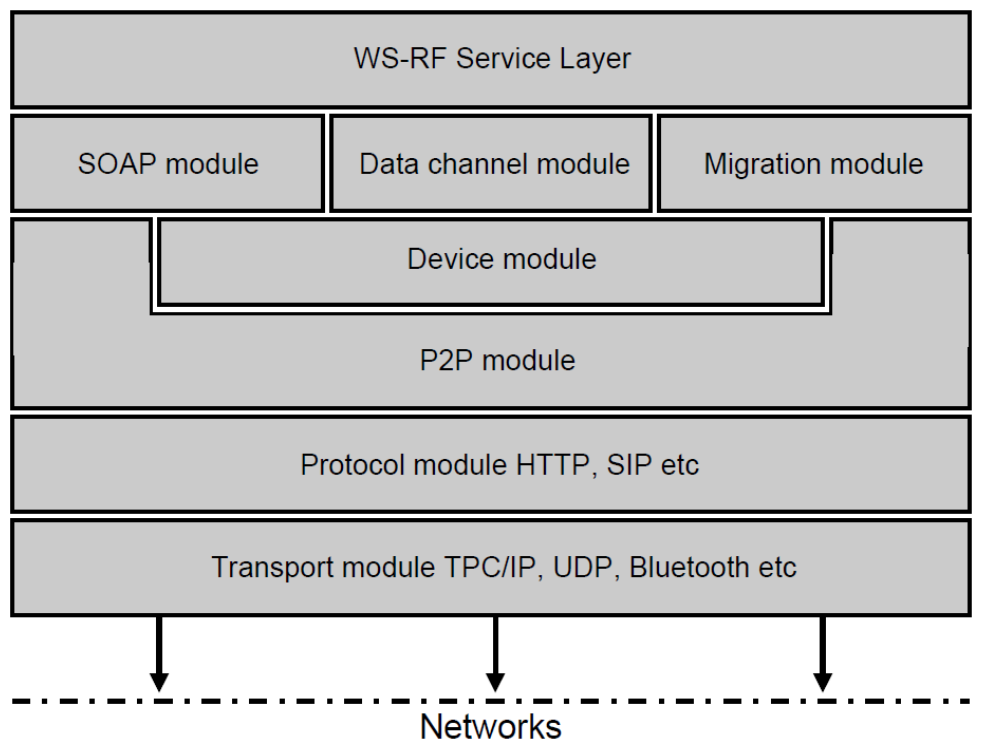

Figure 2. The architectural components of the mobile node[15]

\section{Conclusions}

The proposed distributed mobile architectural design (Mobile P2G) strengthens research efforts in Peer-to-Peer and Grid technologies, addressing issues such as transient device mobility, while supporting interactive component configurability for distributed dynamic scenarios, strongly focused on data transfer and integration.

This process of creating a $\mathrm{P} 2 \mathrm{G}$ hybrid architecture represents a change of vision and a new paradigm from two perspectives: firstly, this hybrid architecture addresses the uniform integration of mobile and static distributed resources by integrating computational Grid and 
www.conferenceie.ase.ro

P2P technologies, incorporating solutions for real mobility and transient connectivity of devices; Secondly, hybrid architecture allows integration of components and data at the time of execution through service-oriented and dynamic discovery of services and resources.

\section{References}

[1] I. Foster, C. Kesselman, J. Nick, and S. Tuecke, "The physiology of the grid: An open grid services architecture for distributed systems integration", Open Grid Service Infrastructure WG, Global Grid Forum, June, 2002.

[2] G. Fox, D. Gannon, and M. Thomas, "A Summary of Grid Computing Environments", Concurrency and Computation: Practice and Experience (Special Issue), 14(13-15):10351044, Jan 2003.

[3] A. Harrison and I. Taylor, "The Web Services Resource Framework In A Peer-To-Peer Context," Journal of Grid Computing, vol. 4, pp. 425-445, December 2006.

[4] A. Kumar and B. Xie, (editors), "Handbook of Mobile Systems Applications and Services", CRC Press, Boca Raton, USA, 2012, Available: https://books.google.ro/books?isbn=1439801533.

[5] P. Kumar and G. C. Deka, "Design and Use of Virtualization Technology in Cloud Computing”, ISBN13: 9781522527855, August 2017.

[6] G. von Laszewski and K. Amin. "Grid Middleware. Middleware for Communications" in Middleware for Communications, edited by Q.H. Mahmoud, Available: http://citeseerx.ist.psu.edu/viewdoc/download?doi=10.1.1.462.3579\&rep=rep1\&type=pdf

[7] E. Udoh and F. Z. Wang, "Handbook of Research on Grid Technologies and Utility Computing: Concepts for Managing Large-Scale Applications", ISBN13: 9781605661841, May 2009.

[8] I. Wang, "P2PS (Peer-to-Peer Simplified)", in Proceedings of 13th Annual Mardi Gras Conference - Frontiers of Grid Applications and Technologies, pp. 54-59, Louisiana, State University, February 2005.

[9] "Dynamic Data Driven Application Simulations", Available at: http://www.dddas.org/.

[10] "The Foundation for Intelligent Physical Agents.” See http://www.fipa.org.

[11] "P2PS - Peer-to-Peer Simplified", Available at: http://www.trianacode.org/p2ps/.

[12] "SLP, Service Location Protocol", Version 2, Available at: http://www.ietf.org/rfc/rfc2608.txt.

[13] "The AKOGRIMO Integrated Project", Available at: http://www.akogrimo.org.

[14] "UDDI Spec TC, UDDI Version 3.0.2", Available at: http://uddi.org/pubs/uddiv3.html.

[15] A. Harrison, I. Kelley, E. Mieilica, A. Ripoșan, I. Taylor, " Mobile Peer-To-Grid Architecture for Paramedical Emergency Operations", IOS Press, 2003. 